\title{
Discovering New Ordered Phases of Block Copolymers
}

\author{
Yardena Bohbot-Raviv* and Zhen-Gang Wang ${ }^{\dagger}$ \\ Division of Chemistry and Chemical Engineering, California Institute of Technology, Pasadena, California 91125
}

(Received 30 March 2000)

\begin{abstract}
We propose a new and general method for discovering novel ordered phases of block copolymer melts. The method involves minimizing a free energy functional in an arbitrary unit cell with respect to the composition profile and the dimensions of the unit cell, without any prior assumption of the microphase symmetry. Varying the initial conditions allows to search for different stable and metastable structures. Application of this method to $A B C$ star and linear triblock copolymers using an approximate free energy reveals new morphologies not yet observed in experiment.
\end{abstract}

PACS numbers: $61.25 . \mathrm{Hq}, 61.41 .+\mathrm{e}, 64.75 .+\mathrm{g}$

Block copolymers serve as a fertile source of "soft materials" exhibiting fascinating periodically ordered microphases such as the "knitting pattern" (KP) [1] and the core-shell bicontinuous gyroid phase [2]. The spontaneous microphase ordering of block copolymers is driven by repulsive interactions acting between distinct blocks, delicately balanced by the configurational entropy which reflects the elasticity of the polymer chain. The chemical bonds connecting different types of blocks keep the segregation on a molecular scale leading to domains of the order of 10-100 nm. The morphology formed in the ordered state depends on composition, the interaction energies between distinct blocks, as well as the particular molecular architecture. These facts are responsible for the rich and complex phase diagrams characterizing block copolymer melts [3].

Different levels of mean field theory have proven to capture the essential features characterizing microphase ordering of block copolymers [3,4]. Among those, the most accurate calculation requires a full self-consistent field (SCF) approach [5]. However, in spite of the theoretical successes, novel morphologies have almost always been discovered first in experiments. The reason is that theoretical approaches are all based upon calculating and comparing free energies of predetermined symmetries. This limitation is especially accentuated when exploring the large parameter space and various molecular designs of multiblock copolymers. On the other hand, Monte Carlo simulation methods, though free of assumptions about the symmetry of the phases, are computationally expensive and limited by finite size effects [6,7].

In this paper we propose an alternative approach for studying the morphology of multiblock copolymers without the need to assume the symmetry of the ordered phase. Drolet and Fredrickson [8] have recently proposed a numerical implementation of SCF theory that, similarly, does not require the assumption of the microphase symmetry. Unlike Matsen and Schick [5] they search for low free energy solutions by solving the self-consistent equations in real space. In contrast to their optimization procedure, our approach involves minimizing an approximate free energy functional in an arbitrary unit cell, with respect to both the density profile and the dimensions of the unit cell. Obviously, the same idea can be implemented using a SCF free energy; however, considerations for computational efficiency led us to propose an approximate free energy which is presented next. We then demonstrate the efficacy of our approach by applying it to $A B C$ star and linear triblock copolymers.

The free energy of an incompressible block copolymer melt, consisting of $n$ polymers in a volume $V$, can in principle be expressed relative to any arbitrary reference free energy such that

$$
F\left[\left\{\Delta \phi_{\alpha}(\vec{r})\right\}\right]=F_{\text {ref }}+\Delta F .
$$

Here $\Delta \phi_{\alpha}(\vec{r})=\phi_{\alpha}(\vec{r})-f_{\alpha}$ is the order parameter of the system. It denotes the local volume fraction of monomers of type $\alpha$, measured relative to its overall (bulk) volume fraction, $f_{\alpha} \equiv N_{\alpha} / N$; where $N_{\alpha}$ is the degree of polymerization of the $\alpha$ block and $N=\sum_{\alpha=A, B, \ldots} N_{\alpha}$ is the overall polymerization index. For simplicity we assume that all monomers occupy the same volume and the different blocks have the same Kuhn length. The reference free energy in (1) is chosen to have the form of a local ideal Flory-Huggins free energy. In terms of the local volume fraction it is given by

$$
F_{\text {ref }}=\frac{n k_{B} T}{V} \sum_{\alpha}^{A, B, \ldots} \int d \vec{r} \frac{\phi_{\alpha}(\vec{r})}{f_{\alpha}} \ln \phi_{\alpha}(\vec{r}) .
$$

As a first approximation for $\Delta F$, we keep terms only up to quadratic power in the order parameter, $\Delta \phi_{\alpha}$. Thus,

$$
\Delta F \simeq \frac{n k_{B} T}{2} \sum_{\alpha, \beta, \vec{k} \neq 0} S_{\alpha \beta}^{-1}(|\vec{k}|) \Delta \phi_{\alpha}(\vec{k}) \Delta \phi_{\beta}(-\vec{k})-F_{\mathrm{ref}}^{(2)},
$$

where $\Delta \phi_{\alpha}(\vec{k})$ is the Fourier transform of the order parameter defined as $\Delta \phi_{\alpha}(\vec{k})=(1 / V) \int d \vec{r} \Delta \phi_{\alpha}(\vec{r}) e^{-i \vec{k} \cdot \vec{r}}$ and $F_{\text {ref }}^{(2)}$ stands for the expression given in (2) expanded up to second order in $\Delta \phi$. The first term in (3) includes contributions from the block copolymer conformational entropy and interaction energy. Within the random phase approximation (RPA) $S_{\alpha \beta}^{-1}=S_{\alpha \beta}^{(o)-1}+\tilde{\chi}_{\alpha \beta}$ where $\tilde{\chi}_{\alpha \beta}=N_{\chi_{\alpha \beta}}$ is the interaction energy between $\alpha$ and $\beta$ monomers expressed in terms of $N$ and a Flory-Huggins 
parameter, $\chi_{\alpha \beta}$, where the interaction energy between similar blocks is chosen to be zero. $S_{\alpha \beta}^{(o)}$ arises from the single (Gaussian) chain density-density correlation function originating from the chain connectivity [9]. Similar approximate free energy has been previously used by several groups for studying the equilibrium microphase ordering of both $A B[10,11]$ and $A B C$ copolymers [12] as well as nonequilibrium [13,14] and surface segregation phenomena [15]. These studies suggest that including the chain architecture at the RPA level captures the essential physics of the problem. Since the present focus is on efficiently discovering new ordered phases, rather than on accurately evaluating their free energy, the use of an approximate free energy is numerically advantageous.

The equilibrium morphology for a given block copolymer melt (i.e., composition and interaction parameters) is found by minimizing the free energy functional (1) subject to the constraint of local incompressibility, $\sum_{\alpha} \Delta \phi_{\alpha}=0$. One way to approach this task in real space involves discretizing the free energy (1). Since we are dealing with periodic structures we focus on an arbitrary unit cell of dimensions $D_{x} D_{y} D_{z}$ and divide it into $M_{x} M_{y} M_{z}$ discrete grid points. In addition to the local composition, the dimensions of the unit cell (i.e., each periodicity $D_{s=x, y, z}$ ) are allowed to vary during the optimization process. In contrast to all previous works that consider minimizing free energy with respect to the periodicity of predetermined symmetries [5,11], our use of an adaptive cell allows the symmetry to be selected by the minimization procedure. On the other hand, our approach is advantageous over straightforward discretizing an arbitrary but fixed volume; it considerably reduces the number of grid points needed and, hence, the equilibration time. More important, it removes any possible constraints imposed by the fixed cell dimension on a periodic microphase, which generally create strains in the system and, thus, bias the system toward structures with lower strain energy. This point has been clearly demonstrated in Monte Carlo simulations of lattice models where it was shown that certain patterns appear only at a particular box size [7].

To demonstrate our approach for exploring new selfassembly we have chosen to first restrict our study to two-dimension periodic microphases; extension to three dimension is straightforward. The scheme we have used consisted of the following. A two-dimension periodic cell was considered such that $\Delta \phi_{\alpha}\left(q_{x}, q_{y}\right)$ becomes the fast Fourier transform of $\Delta \phi_{\alpha}\left(n_{x}, n_{y}\right)$, where the integer wave number, $q_{s}$, and the integer coordinate number, $n_{s}$, are defined through $k_{s}=2 \pi q_{s} / D_{s}$ and $r_{s}=n_{s} D_{s} / M_{s}(s=x, y)$, respectively. For the initial guess of $\Delta \phi_{\alpha}$ a random configuration (either in real or Fourier space) was constructed within an arbitrary unit cell divided into $M_{x} \times M_{y}$ grid points. For the $\chi N$ values considered here $32 \times 32$ grid points produced an accuracy of at least $10^{-4}$ in the free energy; a finer grid would be required for higher values of $\chi N$. The equilibrium density profile and size of the unit cell were determined by simultaneously solving the set of equations $\left[\partial F / \partial \Delta \phi_{\alpha}\left(n_{s}\right)\right]=0,\left(\partial F / \partial D_{s}\right)=0$, using standard numerical minimization algorithms such as the steepest descent and the conjugate gradient methods. Each minimization was repeated several times and examined using different initial conditions and a different number of grid points. The resulting patterns, presented next in the form of density plots, were constructed using the following color scheme: Three different colors, red, green, and blue were, respectively, assigned to the three $A, B$, and $C$ blocks. Only one color (red, green, or blue), corresponding to the highest value of the local volume fraction, was plotted at each point in the cell, with an intensity proportional to its volume fraction. For a clear presentation of the final pattern, the linear dimensions of the final unit cell were replicated 3 times in each direction. The lengths were scaled by the radius of gyration of an ideal chain, $R_{g}$.

As a first application of our approach we have examined several points in the parameter space of $A B C$ star and linear triblock copolymers. The three density plots shown in Figs. 1(a)-1(c) correspond to symmetric star triblock copolymers (i.e., $f_{A}=f_{B}=f_{C}$ ). A star copolymer melt, with $\tilde{\chi}_{A B}=\tilde{\chi}_{B C}=\tilde{\chi}_{A C}=26$, separated into three microdomains forming a hexagonal (HEX) honeycomb lattice characterized by $D_{y} / D_{x}=1.732052 \simeq \sqrt{3}$ [Fig. 1(a)]. Repeating the calculation for slightly different interaction parameters revealed that this phase is indeed robust. The same morphology has been obtained in threedimension Monte Carlo simulations under similar conditions [7] and was recently obtained in a SCF calculation for a linear $A B C A$ tetrablock copolymer melt [8]. For the same system a metastable structure consisting of hexagonally ordered lozenge-shaped columns was also obtained [Fig. 1(b)]. Both structures have been previously hypothesized for $A B C$ star triblock copolymers [16], although they have yet to be obtained experimentally.

We have studied other types of star triblock microphases by first varying the interaction parameters while keeping $f_{A}=f_{B}=f_{C}$. When $\tilde{\chi}_{A B}<\tilde{\chi}_{A C}=\tilde{\chi}_{B C}=20$ the system converged to a HEX phase, with $C$ blocks comprising the cylinders embedded in a continuous matrix of $A$ and $B$ blocks (not shown). On the other hand, when $\tilde{\chi}_{A B}$ is increased above $\tilde{\chi}_{B C}=\tilde{\chi}_{A C}=23$ a lamellar ordering, composed of a lamella of $C$ blocks and a lamella of alternating $A$ and $B$ cylinders, was obtained [Fig. 1(c)]. Finally, for $\tilde{\chi}_{A B} \ll \tilde{\chi}_{A C}<\tilde{\chi}_{B C} \sim 30$, a HEX phase of $C$ cylinders, within a nonuniform matrix of $A$ and $B$ blocks formed (not shown). Interestingly, breaking the composition symmetry changed the morphology to the core-shell HEX phase shown in Fig. 1(d). Unlike the patterns shown in Figs. 1(a)-1(c), the HEX and core-shell HEX phases [Fig. 1(d)] were both experimentally observed in $A B C$ star triblock copolymer melts [17].

Recent investigations of linear triblock copolymers have led to the discovery of some fascinating two-dimension morphologies such as the KP. In attempting to reproduce 

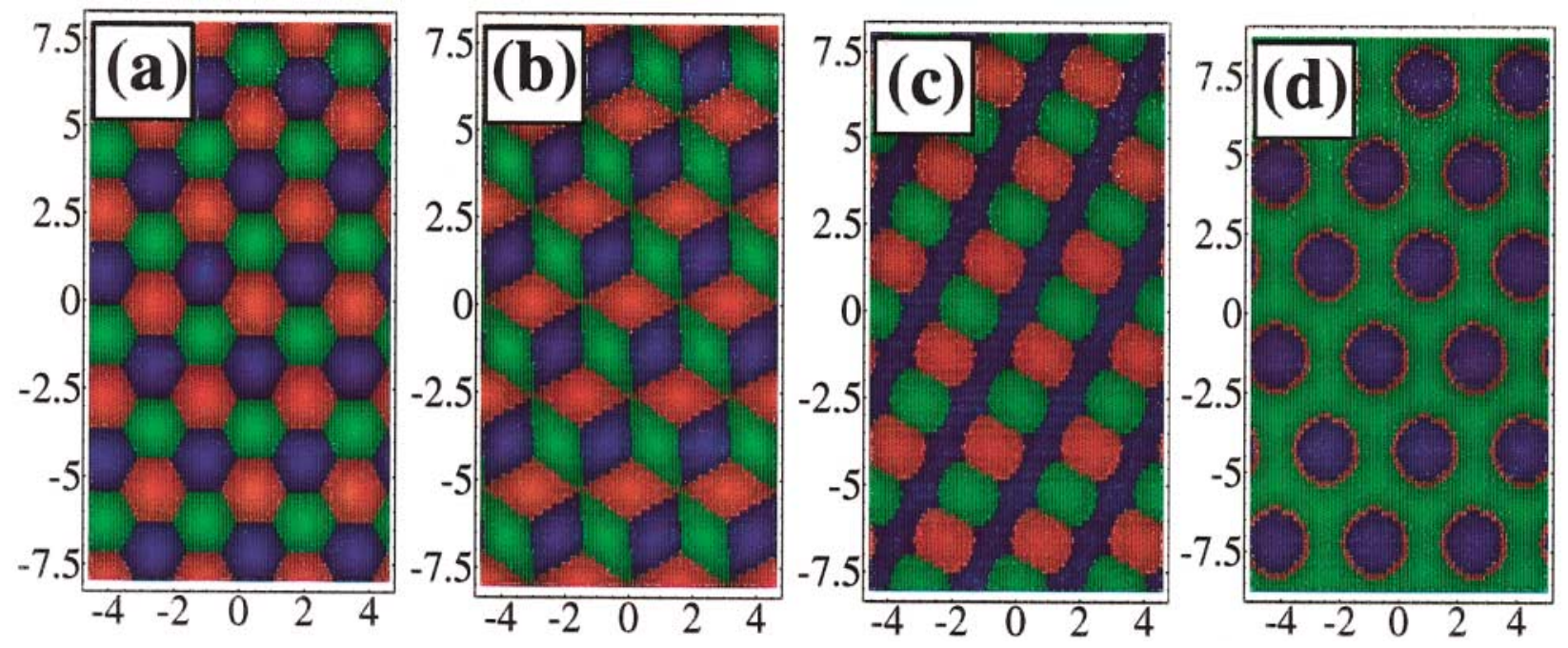

FIG. 1 (color). Ordered microphases obtained using the proposed method and free energy for $A B C$ star triblock copolymers. Domains rich in $A, B$, or $C$ blocks are shown in red, green, or blue, respectively. The color scale is explained in the text. (a),(b) $f_{A}=$ $f_{B}=f_{C}$ and $\tilde{\chi}_{A B}=\tilde{\chi}_{B C}=\tilde{\chi}_{A C}=26$. The free energies per chain in units of $k_{B} T$ are -0.306979 and -0.237951 , respectively. (c) $f_{A}=f_{B}=f_{C}, \tilde{\chi}_{A B}=26$, and $\tilde{\chi}_{B C}=\tilde{\chi}_{A C}=23$. (d) $f_{A}=6 / 24, f_{B}=11 / 24, \tilde{\chi}_{A B}=20, \tilde{\chi}_{B C}=30$, and $\tilde{\chi}_{A C}=28$. The scale in each figure is different.

this phase we have explored regions of parameter space for which the $A B C$ linear triblock copolymer is almost symmetric, i.e., $f_{A}=0.36$ and $f_{C}=0.33$ [1]. The results we present next are obviously preliminary and incomplete mainly due to our restriction to two-dimension structures. Nevertheless, they reveal a series of metastable phases which are amenable to initial conditions, emphasizing the advantage of the method for exploring the relative stability of different phases and possible kinetic pathways. It is possible and indeed likely that some of these metastable phases will become stable in other regions of the parameter space.

Two sets of results, all of which keep the same order of $\tilde{\chi}$ values, $\tilde{\chi}_{A B} \ll \tilde{\chi}_{A C}<\tilde{\chi}_{B C}$, are shown in Figs. 2 and 3. Starting from a random density profile in real space a metastable phase shown in Fig. 2(a) has evolved. Because of its resemblance to the KP [1] we shall denote it as the "knittinglike pattern" (KLP). A metastable lamellacylinder (LC) phase, similar to that shown in Fig. 2(b), but with different relative positions of the cylinders, emerged from a slightly randomized KLP profile. In some cases, depending on the initial degree of randomness, either the KLP was recovered or a stable LC phase [Fig. 2(b)] was obtained. The LC pattern has previously been studied and obtained in experiments of $A B C$ triblock copolymers [18]. Interestingly, at lower values of $\tilde{\chi}$, a random initial profile converged to a "tricolor checkerboardlike" (TCB) phase characterized by $D_{y} / D_{x}=1$ [Fig. 2(c)]. From our preliminary studies of the patterns formed in $A B C$ linear copolymers, the TCB phase was found stable for relatively large regions of parameter space. A similar phase, composed of $A$ and $C$ cylinders tetragonally ordered within a $B$ matrix, has been previously obtained in experiment [19] and studied theoretically [12]. Although the KP was not obtained in this search it was experimentally discovered in the vicinity of the lamella and the LC [Fig. 2(b)] phases [1].

The second interesting set of calculations at higher values of $\tilde{\chi}$ yielded new morphologies from which we present only three of the lowest free energy structures obtained. The microphases shown in Figs. 3(a)-3(c) were each obtained from different initial conditions: a random initial profile constructed in real space, a hexagonal order of $A$ (red) cylinders in a $B / C$ matrix, and a hexagonal order of
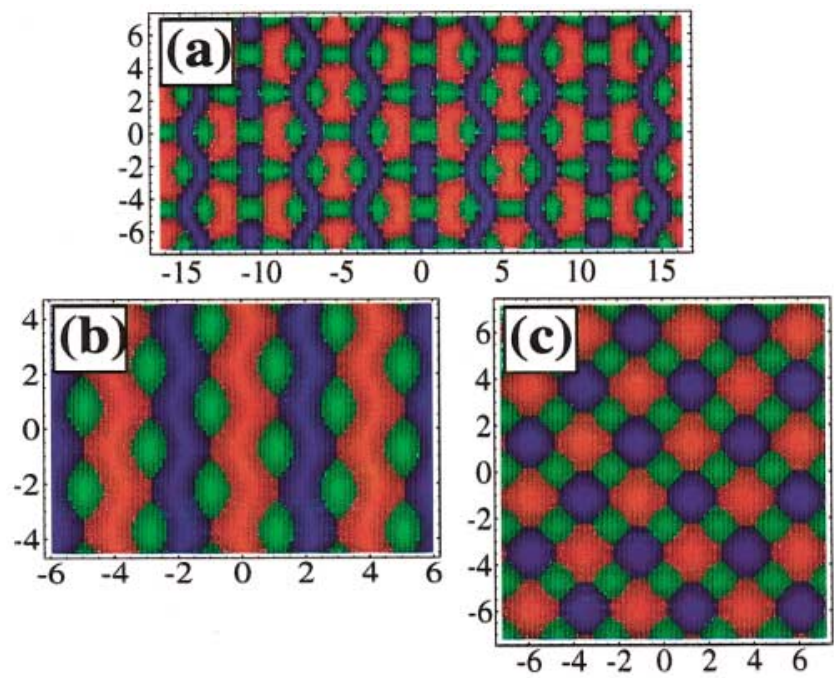

FIG. 2 (color). Ordered microphases of $A B C$ linear triblock copolymers with $f_{A}=0.36$ and $f_{B}=0.31$. (a),(b) $\tilde{\chi}_{A B}=30$, $\tilde{\chi}_{B C}=32$, and $\tilde{\chi}_{A C}=22$; a "knittinglike pattern" (KLP) and a lamella-cylinder (LC) phase. (c) $\tilde{\chi}_{A B}=26, \tilde{\chi}_{B C}=30$, and $\tilde{\chi}_{A C}=20$; a "tricolor checkerboardlike" (TCB) phase. The scale in each figure is different. 


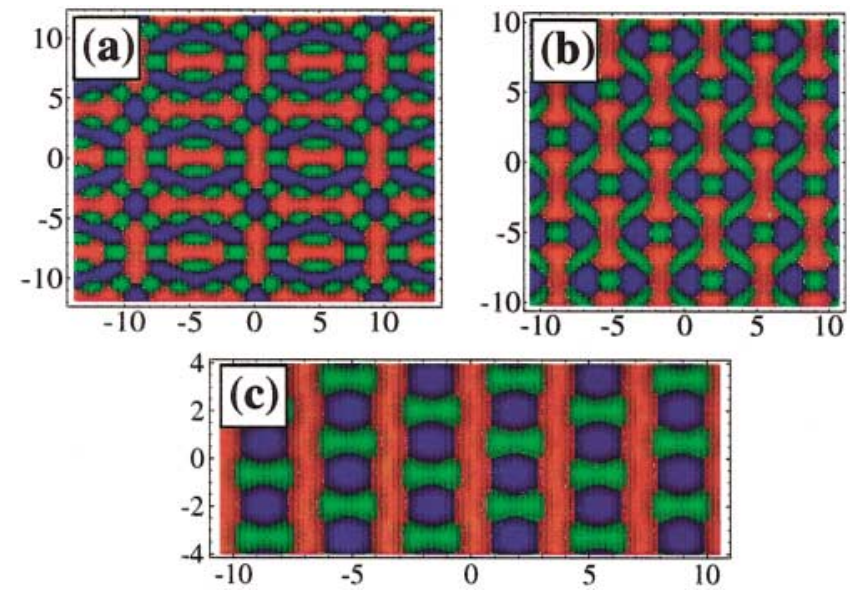

FIG. 3 (color). Ordered microphases of $A B C$ linear triblock copolymers obtained from different initial conditions for $f_{A}=0.36$ and $f_{B}=0.31$. Here, $\tilde{\chi}_{A B}=30, \tilde{\chi}_{B C}=35$, and $\tilde{\chi}_{A C}=22$. (a) An "interconnected wheel-like" (IW) pattern. (b) A decorated lamella-cylinder pattern. (c) An alternating lamella-cylinder pattern. The scale in each figure is different.

$C$ (blue) cylinders in an $A / B$ matrix. The pattern shown in Fig. 3(a) was found to have the lowest free energy. It resembles interconnected wheels (IW) where the center of each wheel (blue) resembles the unit cell of the KP, whereas, each connection resembles the unit cell of the TCB pattern. The second ordered phase shown in Fig. 3(b) has a pattern of alternating layers of $A$ (red $)$ "bones" and $C$ (blue) cylinders, each differently decorated with $B$ (green) blocks. The morphology of the third metastable phase, shown in Fig. 3(c), clearly retained the initial $C$ block (blue) microdomains, from which it is understood that the minimization process mainly involved the separation of $A$ and $B$ blocks. The TCB pattern was also obtained for this system starting from a random profile in Fourier space. However, its free energy lies in between that of the patterns shown in Figs. 3(a) and 3(c). The susceptibility of the final phase to different initial conditions, as featured in these examples, is essential for discovering various stable and metastable phases and can be exploited to study metastability arising from different degrees of preordering constraints.

In this paper we have proposed a new method for discovering ordered phases of block copolymer melts. The central idea is based on discretizing and minimizing a free energy functional in an arbitrary unit cell in real space, and allowing for both the profile and dimensions of the unit cell to vary. Application of the method and its efficacy to predicting two-dimension ordered phases have been demonstrated using an approximate free energy for triblock copolymer melts. Several new stable and metastable morphologies of triblock copolymer melts have been revealed, reflecting the ability of the method to explore the diverse self-assembly of multiblock copolymers and to help guide experimental design of new mate- rials. To effectively search the vast configuration space of multiblock copolymers more ellaborated sampling methods such as simulated annealing or parallel tempering [20] may be considered. A powerful extension of our approach is to combine it with SCF, wherein new ordered morphologies identified by our approach serve as input for more elaborated SCF calculations. This combination will provide the long desired theoretical tool for efficiently and accurately predicting, a priori, complex phase diagrams of multiblock copolymer melts.

We acknowledge financial support from the National Science Foundation, the Camille and Henry Dreyfus Foundation, and the Alfred P. Sloan Foundation.

*Electronic address: yarden@caltech.edu

†Electronic address: zgw@cheme.caltech.edu

[1] U. Breiner, U. Krappe, E. L. Thomas, and R. Stadler, Macromolecules 31, 135 (1998).

[2] T. Goldacker and V. Abetz, Macromolecules 32, 5165 (1999); T. A. Shefelbine et al., J. Am. Chem. Soc. 121, 8457 (1999); Y. Matsushita, J. Suzuki, and M. Seki, Physica (Amsterdam) 248B, 238 (1998).

[3] F. S. Bates and G. H. Fredrickson, Phys. Today 52, No. 2, 32 (1999).

[4] M. W. Matsen and F. S. Bates, Macromolecules 29, 1091 (1996).

[5] M. W. Matsen and M. Schick, Phys. Rev. Lett. 72, 2660 (1994).

[6] K. Binder, in Advances in Polymer Science, edited by A. Abe et al. (Springer-Verlag, Berlin, 1994), Vol. 112; R. G. Larson, J. Phys. II (France) 6, 1441 (1996).

[7] T. Dotera and A. Hatano, J. Chem. Phys. 105, 8413 (1996).

[8] F. Drolet and G. H. Fredrickson, Phys. Rev. Lett. 83, 4317 (1999).

[9] L. Leibler, Macromolecules 13, 1602 (1980).

[10] T. Ohta and K. Kawasaki, Macromolecules 19, 2621 (1986).

[11] J. Melenkevitz and M. Muthukumar, Macromolecules 24, 4199 (1991); R. L. Lescanec and M. Muthukumar, Macromolecules 26, 3908 (1993).

[12] H. Nakazawa and T. Ohta, Macromolecules 26, 5503 (1993); W. Zheng and Z.-G. Wang, Macromolecules 28, 7215 (1995).

[13] S. Qi and Z.-G. Wang, J. Chem. Phys. 111, 10681 (1999).

[14] Y. Oono and Y. Shiwa, Mod. Phys. Lett. B 1, 49 (1987).

[15] H. Chen and A. Chakrabarti, J. Chem. Phys. 108, 6897 (1998).

[16] S. Okamoto et al., Polymer 38, 5275 (1997).

[17] S. Sioula, N. Hadjichristidis, and E. L. Thomas, Macromolecules 31, 5272 (1998); N. Hadjichristidis et al., Macromolecules 26, 5812 (1993).

[18] R. Stadler et al., Macromolecules 28, 3080 (1995).

[19] Y. Mogi et al., Macromolecules 25, 5408 (1992).

[20] S. Kirkpatrick, C. D. Gelatt, and M. P. Vecchi, Science 220, 671 (1983); C. J. Geyer and E. A. Thompson, J. Am. Stat. Assoc. 90, 909 (1995). 